\title{
THE RESEARCH OF SPECTRAL RECONSTRUCTION FOR LARGE APERTURE STATIC IMAGING SPECTROMETER
}

\author{
Huaquan Lv ${ }^{1}$, Yaran Lee ${ }^{1, *}$, Rundong Liu ${ }^{1}$, Chengcheng Fan ${ }^{1}$, Youju Huang ${ }^{1}$ \\ ${ }^{1}$ Guangxi Zhuang Autonomous Region Institute of Remote Sensing Information Surveying and Mapping, 530023 Nanning, China - \\ lyr19930813@163.com
}

Commission VI, WG III/4

KEY WORDS: Interference Imaging Spectroscopy, Apodization, Phase Correction, Fourier Transform, Spectral Reconstruction

\begin{abstract}
:
Imaging spectrometer obtains or indirectly obtains the spectral information of the ground surface feature while obtaining the target image, which makes the imaging spectroscopy has a prominent advantage in fine characterization of terrain features, and is of great significance for the study of geoscience and other related disciplines. Since the interference data obtained by interferometric imaging spectrometer is intermediate data, which must be reconstructed to achieve the high quality spectral data and finally used by users. The difficulty to restrict the application of interferometric imaging spectroscopy is to reconstruct the spectrum accurately. Based on the original image acquired by Large Aperture Static Imaging Spectrometer as the input, this experiment selected the pixel that is identified as crop by artificial recognition, extract and preprocess the interferogram to recovery the corresponding spectrum of this pixel. The result shows that the restructured spectrum formed a small crest near the wavelength of $0.55 \mu \mathrm{m}$ with obvious troughs on both sides. The relative reflection intensity of the restructured spectrum rises abruptly at the wavelength around $0.7 \mu \mathrm{m}$, forming a steep slope. All these characteristics are similar with the spectral reflection curve of healthy green plants. It can be concluded that the experimental result is consistent with the visual interpretation results, thus validating the effectiveness of the scheme for interferometric imaging spectrum reconstruction proposed in this paper.
\end{abstract}

\section{INTRODUCTION}

Imaging spectroscopy is a new type of remote sensing observation developed in the 1980s. By combining imaging technology with spectroscopy, it can obtain or indirectly obtain the spectrum data characterizing the physical properties of the target while acquiring the target image. Imaging spectroscopy can obtain remote sensing images containing tens or even hundreds of narrow and continuous bands with spectral resolution up to nanoscale, which provides a powerful means for material analysis and identification, and has important application value in military, geological survey, environmental monitoring and precision agriculture, is becoming the development trend of modern remote sensing technology.

Interferometric imaging spectrometer is based on the principle of light interference, and the modulated spectral information is reflected by the interference fringes superimposed on the target image. The time-modulated interferometric imaging spectrometer relies on moving mirror scanning to form the optical path difference. Its technical implementation is difficult, and the shortcomings of poor aseismic performance on the satellite and inability to acquire spectral information in real time also limit its application. In contrast, the spatial-modulated interferometric imaging spectrometer does not require highprecision moving mirror components and overcomes the shortcomings of time-modulated interferometric imaging spectrometer. However, the presence of incident slit reduces the luminous flux entering the system, although it does not affect the spectral resolution, but it limits the spatial resolution. Large Aperture Static Imaging Spectrometer(LASIS) adopts time- space combined modulation mode, does not have an incident slit, and does not rely on moving parts to acquire spectral information. Array detector provides the interference image of instantaneous ground view field, so the complete interferogram of the ground features is obtained by push the whole field of view. The technical difficulties that restrict the application of LASIS are the accurate acquisition of interferograms and accurate reconstruction of spectrum.

\section{SPECTRAL RECONSTRUCTION}

Since Large Aperture Static Imaging Spectrometer directly obtains the interference data of target, it is intermediate data, which must be processed by spectral reconstruction to acquire high-quality spectral data, and ultimately used by the user. Spectral reconstruction is a necessary step in the application of interferometric imaging spectroscopy, and the pros and cons of the reconstruction results directly constrain the application value of subsequent spectral information.

Interferometric imaging spectral reconstruction takes the interference data obtained by instrument as input, and its processing flow mainly includes interference data preprocessing and spectral reconstruction processing.

Interference data preprocessing is mainly used to correct errors and improve the precision of reconstructed spectrum. Due to the impact of instrument itself and working environment, it is unavoidable to introduce various deviations in the process of obtaining interference images. But even very subtle errors in the interferogram may cause large distortions in the reconstructed 
spectrum. Therefore, it is difficult to satisfy the application requirements of high-precision spectral data only by performing Fourier integral transform on interference data, and various correction methods are applied to the interference data preprocessing.

Spectral reconstruction enables the conversion from interference data to the final target spectrum, thus ensuring the successful application of the interference data.

\section{EXPERIMENTS AND ANALYSIS}

In this paper, the hyperspectral images obtained by Yaogan-14 satellite are used as experimental data to study the related processes of spectral reconstruction. The interferogram of any pixel in the interference image is processed by spectral reconstruction to acquire the corresponding spectral curve. The same process is repeated for interferograms of all pixels, and then a complete physical hyperspectral image sequence can be obtained by splicing. So the experiments in this paper starts with the interferogram of single pixel.

\subsection{Experimental Data}

Yaogan-14 satellite is working in the $479 \mathrm{~km}$ sun synchronous orbit, using two sets of imaging spectrometer (visible light imaging spectrometer and infrared imaging spectrometer) to complete the detection task. Its ground resolution are $10 \mathrm{~m}$ and $20 \mathrm{~m}$, the number of channels are 180 and 256 , which covers the spectral range of 0.45 to 2.5 microns (visible light: 0.456 0.918 micron; infrared: $0.881 \sim 2.529 \mathrm{~m}$ ).

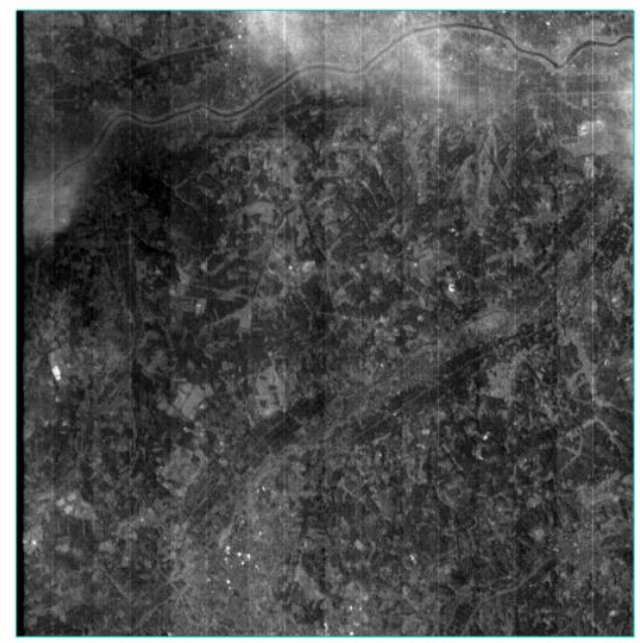

Figure 1. Yaogan-14 satellite original image

Figure 1 above shows the original image used in the experiment The photographed area is located in Misawa City, Japan, and the imaging time is 2014-05-28T 12:50. Misawa City is situated in the northeastern part of the Kanto Plain. The terrain is mostly hilly and lowland, with a maximum elevation of $57 \mathrm{~m}$. It is a temperate marine monsoon climate with an average annual temperature of about $13^{\circ} \mathrm{C}$ and an annual rainfall of 1300 to 1600 millimeters.

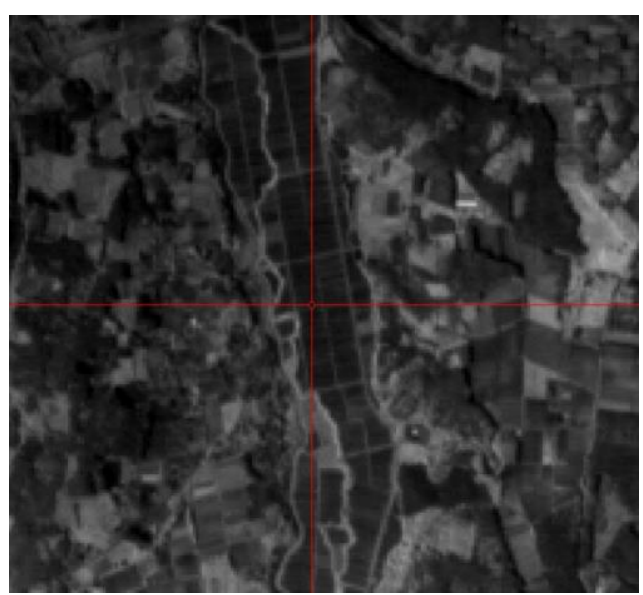

Figure 2. Experimental sample (crops) schematic

In this chapter, the pixel of 423rd rows and 184th columns in the original image is used as experimental sample. As shown in Figure 2, it can be determined visually that the features at the point are crops.

\subsection{Interferogram removal of DC component and trend items}

Under ideal circumstances, the interferogram extracted based on a single ground pixel should be the oscillation of the intensity centered on the zero, and the interference intensity gradually decreases with the increase of the optical path difference, which is similar to the sinusoidal distribution curve. The purpose of removing the DC component is to make the interferogram as close to its ideal distribution as possible, which is also the basis for judging the effect of DC removal. Figure 3 is shown as the original interference curve extracted from the experimental sample.

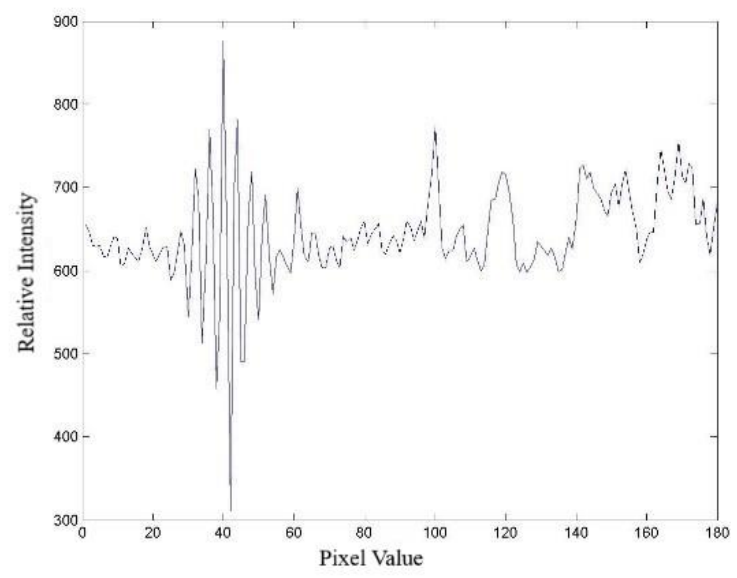

Figure 3. Original interferogram

It can be clearly seen that there exists a direct current component with an intensity value around 620 in the original interference diagram, and the spectral information is mainly contained in the alternating current component of the interference curve. That is, the direct current component only serves as a background component of the interferogram and has no meaning. Its existence will make mistakes in subsequent 
processing steps, so it needs to be removed. Since Yaogan-14 satellite adopts the Large Aperture Static Imaging Spectrometer technology scheme and relies on the push-broom of array detector to obtain interference data, so the interferogram is sampled at equal intervals. This chapter uses the following formula (1) to remove the DC component:

$$
\mathrm{I}^{\prime}(\mathrm{i} \cdot \delta \Delta)=\mathrm{I}(\mathrm{i} \cdot \delta \Delta)-\mathrm{C}=\mathrm{I}(\mathrm{i} \cdot \delta \Delta)-\frac{1}{\mathrm{~N}} \sum_{\mathrm{i}=1}^{\mathrm{N}} \mathrm{I}(\mathrm{i} \cdot \delta \Delta)
$$

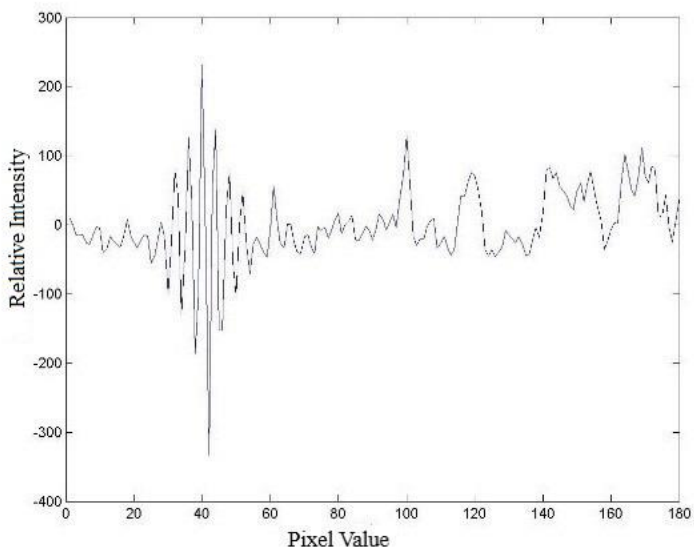

Figure 4. Remove the DC component

Observing the interferogram after removing the DC component, it is clear that there is a tendency for the interference curve to rise slowly as ttyhe pixel value increases. The trend term is a frequency component that has a vibration period longer than the signal sampling length, and is generally caused by improper processing of the original signal in the sampling process. The existence of trend error will have a great impact on the spectrum subsequently reconstructed, so measures must be taken to eliminate it. By analyzing the primitive interferograms of multiple pixels, it is found that their trend trends are quite different. Therefore, in order to achieve better trend term elimination effects when interferograms are preprocessed, we cannot simply regard it as a linear trend.

There are many methods that can be used to eliminate trend items, depending on the physical model of the test object, signal characteristics and other factors. This chapter uses the least squares method to eliminate the trend terms of the interferograms. First, a general polynomial model of the trend term is established according to the least squares principle, and the curve of the fitted polynomial is subtracted from the original interferogram, thereby eliminating the nonlinear trend terms in the signal.

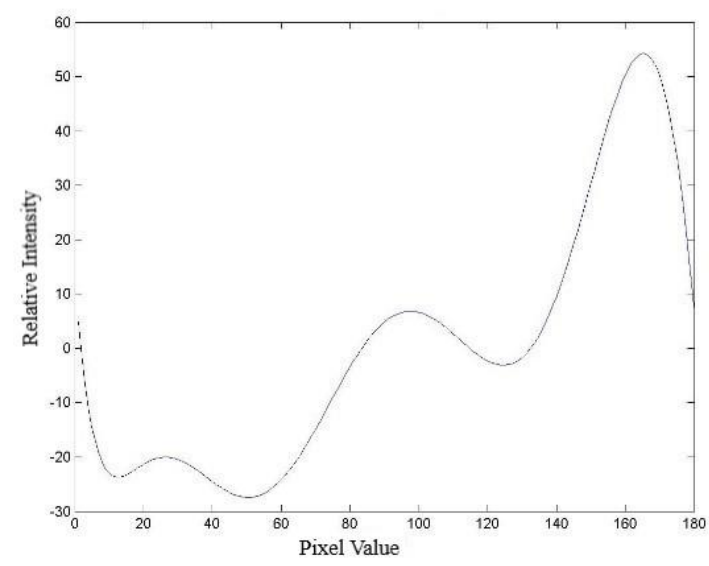

Figure 5. Eight-order nonlinear trend terms

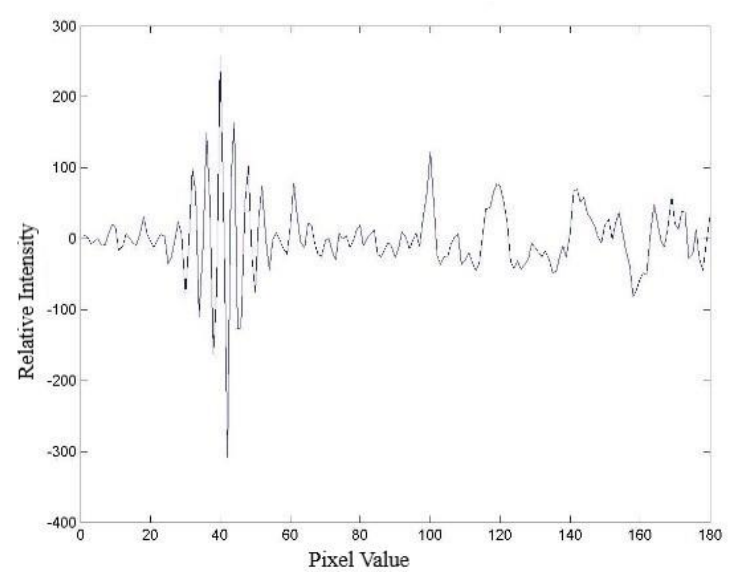

Figure 6. Remove the trend items

In the experiment, the general polynomial models obtained by fitting different orders and the elimination results of their respective trend terms are compared and analyzed. Finally, an eight-order nonlinear trend term that can reflect the variation trend of interference intensity is adopted. Figure 6 shows the interferogram after removing DC components and trend terms. It can be seen that the interference curve after the elimination of the trend terms using the least square method is closer to the ideal degree.

\subsection{Apodization}

The interference intensity plunged to zero outside the maximum optical path difference interval in the actual measurement. In order to reduce or eliminate the spectral energy leakage due to the sudden truncation of the interference curve, different functions can be used to truncate the interference signal. This truncation function is also known as apodization function or window function. The effect of different apodization functions on the signal spectrum is also different. In actual operation, the apodization function selection should consider its effect on spectral reconstruction and apodization effect. That is, the main lobe of the linear function of the instrument should be as narrow as possible and the side lobe should be as low as possible. 
This experiment used the four functions of triangular window, Hanning window, Hamming window, and Blackman window to apodize the data obtained in the previous section, and do a Fourier transform to compare them. The apodization effect of the four window functions and its effect on the full width at half maximum show that the experimental results obtained by the Hanning window function are best.

Figure 7 shows the comparison of the results before and after apodization of the original interference data. The blue curve represents the interferogram without apodization, and the red curve represents the interferogram after apodization.

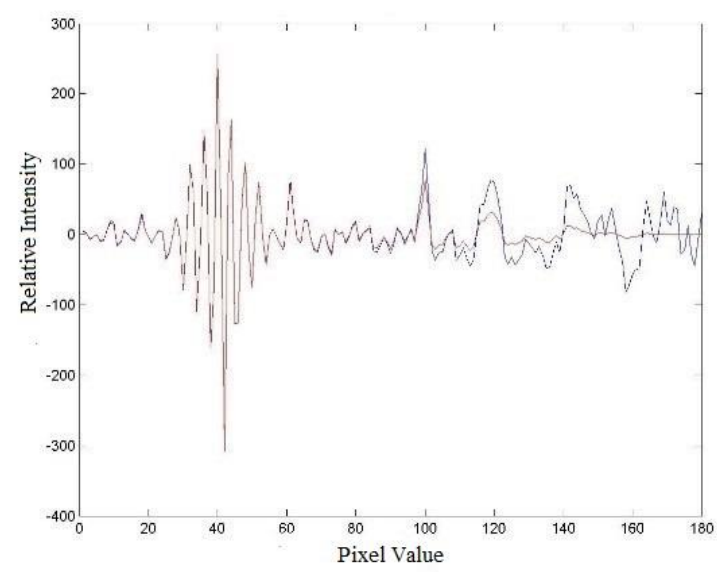

Figure 7. Comparison of results before and after apodization

\subsection{Phase Correction}

Ideally, the interferogram obtained by the interference imaging spectrometer should be completely symmetric about the zero path difference, and Fourier integral transform can be used to determine the spectral curve of the corresponding object. However, in practical engineering, due to the influence of objective factors such as poor uniformity of the beam splitter and unequal sampling interval, there are usually zero-drift and asymmetry between interferometric data collected by detectors, which are classified as phase errors. In order to accurately acquire the spectral information of the object, it is necessary to first correct the phase error before performing the Fourier transform on the interferometric data.

The phase correction first needs to correct the offset of the zero path difference of the interference data, which is essentially equivalent to the accurate selection of the zero path difference position. The sampling point near the zero path difference of interferogram contains the low frequency components of spectral information, and determines the overall trend of the recovery spectrum. Therefore, the position error of zero optical path difference will bring great influence to the result of spectral reconstruction.

According to the principle of interference imaging spectroscopy, the zero optical path difference is the center of the symmetry of the interferogram, receiving the coherent information of two parallel beams with zero optical path difference. The image is modulated to the highest degree and the interference fringes are the most obvious. Randomly select multiple pixels of experimental data and extract their original interferograms for analysis, We found that the center of symmetry is always located at forty-second pixels, and the interference intensity reaches the minimum value at this pixel. It can be determined that the zero optical path difference is located in the fortysecond pixel of the interferogram, rather than the fortieth pixel indicated by the instrument. The subsequent processing steps are carried out with forty-second pixel points as the position of zero optical path difference.

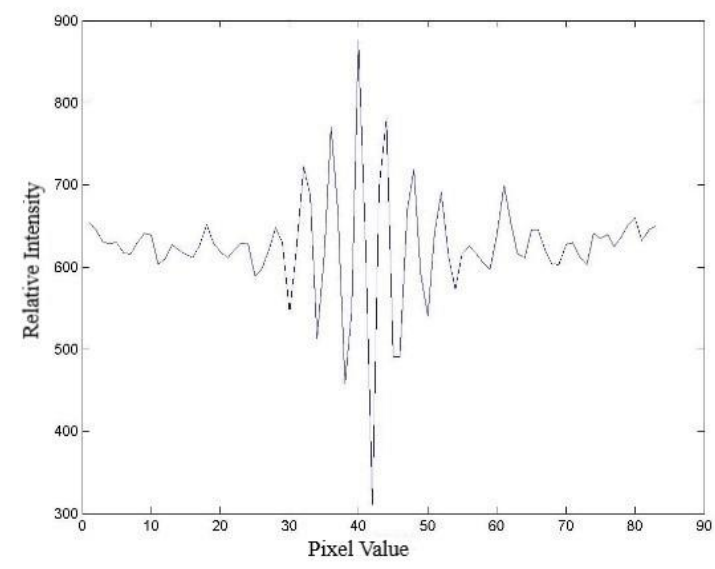

Figure 8. Small bilateral interference data

The experimental data in this chapter sampled the interference information by zero-cross sampling. That is, by collecting a part of data on the other side of the unilateral interferogram, a small bilateral interference diagram with zero optical path difference as the center is constructed. According to the principle of interferometric imaging spectroscopy, the sampling point near the zero optical path difference position determines the overall shape of the reconstructed spectrum, and the sampling point near the maximum optical path difference determines the detailed change of the reconstructed spectrum, as shown in Figure 8 for selecting small bilateral interference data and the zero-cross sampling interference data were separately subjected to a Fourier transform to recover the spectrum. That is to say, the spectral information recovered by using the small bilateral interference patterns also covers the desired spectral band, but the accuracy of the recovered spectrum is poor compared to the bilateral interferogram. Since the small bilateral interferogram contains all the phase information, the corresponding phase offset can be extracted from the small bilateral asymmetry when performing phase correction on the interferometric data. 

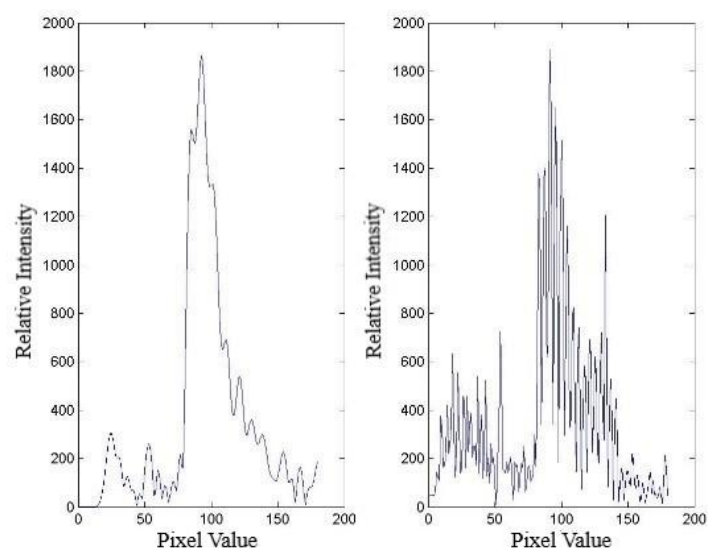

Figure 9. Fourier transform of small bilateral interference data and zero sampling interference data

The correction of nonlinear phase errors needs to be realized by numerical calculation.

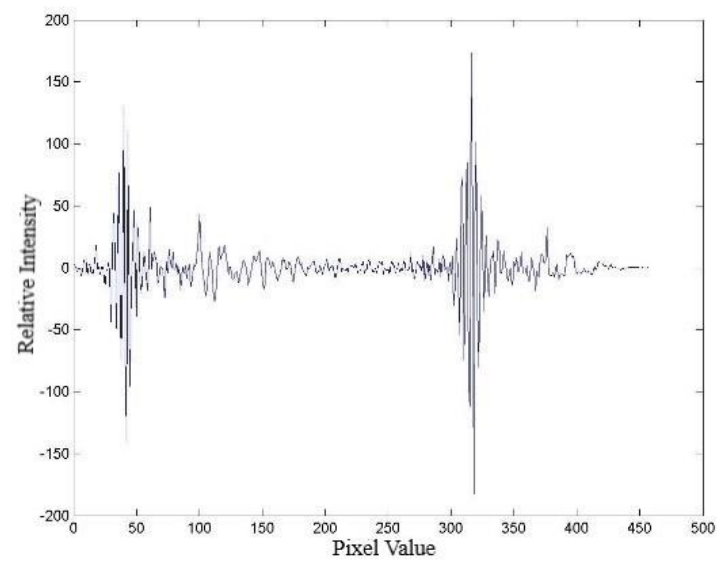

Figure 10. Phase correction interferogram

In theory, the imaginary part of the reconstructed spectral data after phase correction should be zero. However, in this experiment, the result obtained after one correction is inconsistent with the theory, and it can be said that the phase correction is not thorough, and the above correction process needs to be repeated until the phase error is sufficiently small. In this chapter, the accuracy of the phase correction process is determined by comparing the difference between the real value and the module value of the reconstructed spectrum after phase correction. If the difference between the two is small, the phase offset is considered to have been limited to a very small range. The purpose of phase error has been reached.

\subsection{Fourier Transform}

After the above pretreatment process for interference data, a more accurate interferogram is acquired, and Fourier transform is applied to obtain the desired target spectrum. Figure 11 below shows the spectral intensity distribution curve obtained after the experimental data was spectrally reconstructed.

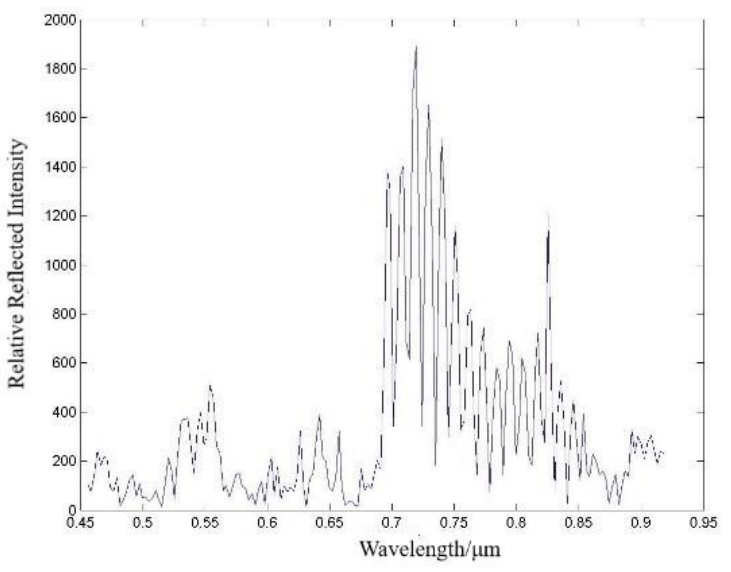

Figure 11. Reconstructed spectrum

\subsection{Analysis for the effectiveness of spectral reconstruction}

The reflection spectrum curve of the feature is used to characterize its ability to reflect electromagnetic radiation as a function of wavelength. The properties of different properties are also different from the reflectance spectra. The reflectance spectrum curve is similar in the same property, but with difference in the water content, surface structure, composition and so on, the spectral curves are also different. Remote sensing is to obtain and record the information of electromagnetic waves reflected by different ground objects in different bands and analyze the differences to identify the attributes of ground objects.

For healthy green plants, the spectral curve always shows a distinct "peak and trough" characteristic, as shown in Figure 12 as the spectral reflectance curve of healthy green plants.

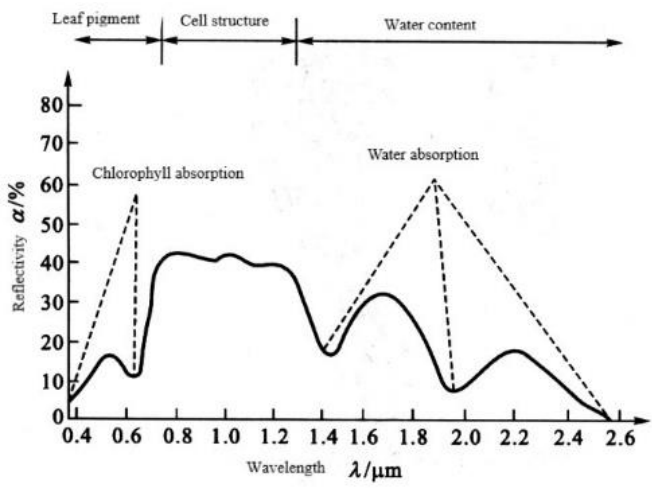

Figure 12. Spectral reflectance curves of healthy green plants

In the visible light range, pigments are the main factors that dominate the spectral response of plants, in which chlorophyll plays the most important role. The spectral curve of a healthy green plant has a small reflection peak near $0.55 \mu \mathrm{m}$ (green), which is the reason for plants to appear green. There are two absorption bands at $0.45 \mu \mathrm{m}$ (blue) and $0.67 \mu \mathrm{m}$ (red) on both sides. There is a steep slope in the near-infrared band between 0.7 and 0.8 microns, and the reflectivity rises sharply, known as the "red edge" phenomenon. This is a plant-specific spectral feature and is a key spectral region of the study. 
The qualitative analysis of the spectral curve reconstructed in the previous section showed a small peak near $0.55 \mu \mathrm{m}$ with a pronounced valley on both sides; the relative reflection intensity of the reconstructed spectrum near $0.7 \mu \mathrm{m}$ suddenly rose to form a steep slope. The above characteristics of the reconstructed spectrum of this experiment are all consistent with the spectral reflectance curve of healthy green plants, and it can be concluded that the features at the pixel point are plants, which is consistent with the result of visual discrimination.

At the same time, the relative reflection intensity of the reconstructed spectrum of this experiment showed a trend of slow decline around $0.73 \mu \mathrm{m}$, which is contrary to the trend of the spectrum curve of healthy green plants. When the plant growth status changes, the shape of its spectral curve will also change, such as plant diseases and pests, or when crops grow poorly due to lack of nutrients and water, the near-infrared peaks will be reduced or even disappear. Based on this, it can be inferred that there may be problems with the crops in the area, and the growth status is poor.

In order to verify the effectiveness of the proposed reconstruction scheme for LASIS, this experiment selected the pixels whose visual discrimination results were rocks, extracted the interferogram and performed the above spectrum reconstruction process. The other experimental sample selected is shown in Figure 13 below.

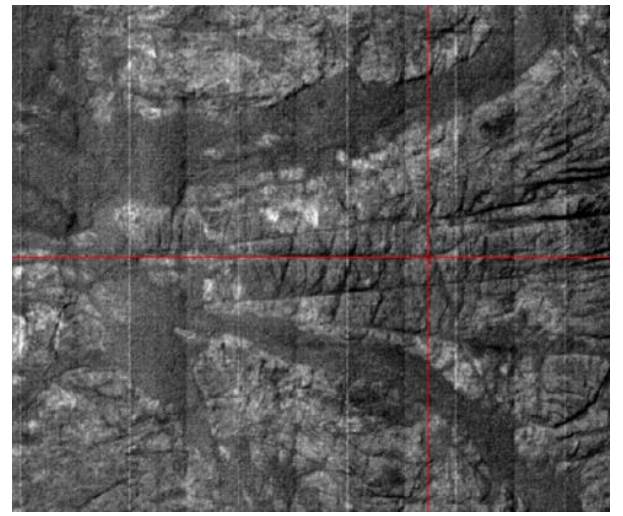

Figure 13. Experimental sample (rocks) schematic

The spectral curve reconstructed by the visual discriminant results for the pixels of the rocks is shown in Figure 14 below.

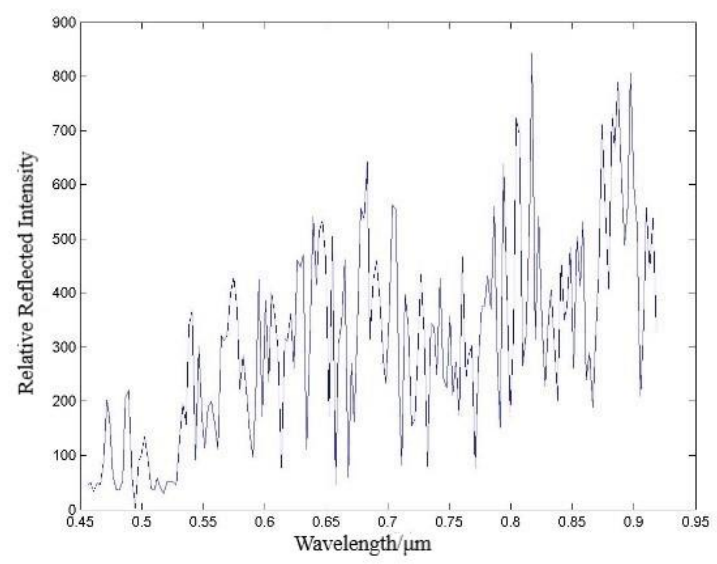

Figure 14. Reconstructed spectrum (rocks)

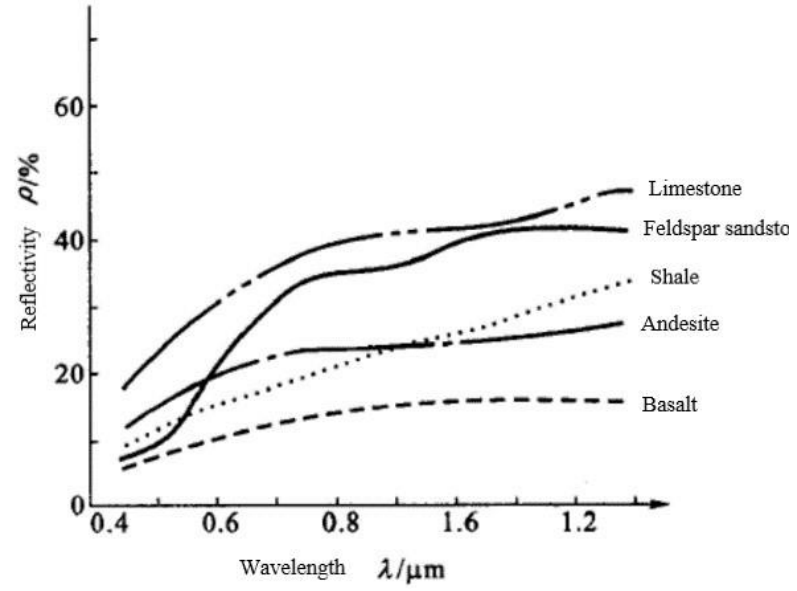

Figure 15. Spectral reflectance curves of rocks

As shown in Figure 15, there is no uniform characteristic of the spectral reflection curve of the rock, and the mineral composition, mineral content, weathering degree, surface smoothness, color and other factors will affect it. Taken together, the spectral reflection curve of the rock slowly and smoothly increases with increasing wavelength, and the experimentally reconstructed spectral curve is consistent with its trend.

This section qualitatively analyzes the reconstructed spectrum of two experimental sample points (crops and rocks) and draws conclusions, verifying the effectiveness of the proposed interference imaging spectral recovery scheme.

\section{CONCLUSION}

Imaging spectroscopy is a powerful tool for mankind to study outer space and the earth itself, and has great potential for application. In this paper, the raw data acquired by the Large Aperture Static Imaging Spectrometer mounted on the Yaogan14 satellite is taken as the experimental input. The theory and implementation method of interference imaging spectral reconstruction are studied.

The original image acquired by Yaogan-14 satellite was used as the experimental input, extracted the interferogram to perform preprocessing and spectral reconstruction processing, and obtained the corresponding recovery spectrum. The experimental results were qualitatively analyzed, verified the interference imaging spectral reconstruction scheme proposed in this paper is effective. In the experiment process, the algorithm is discussed and optimized according to the characteristics of the data. The apodization effect of different window functions and its effect on the actual restoration spectrum are studied in detail. Different correction methods are proposed for linear phase error and nonlinear phase error respectively.

The analysis of the recovery spectrum curve in this paper is limited to simple qualitative understanding and lack of quantitative and in-depth analysis. The research of spectrum matching technology based on hyperspectral database is of great significance for promoting the application and development of imaging spectroscopy technology. 


\section{REFERENCES}

Bennett C L, Carter M R, Fields D J, et al. Imaging Fourier transform spectrometer (LIFTIRS)[J]. Proceedings of SPIE The International Society for Optical Engineering, 1993, 1937:191-200.

Breckinridge J B. Evolution of imaging spectrometry: past, present, and future[J]. Proceedings of SPIE - The International Society for Optical Engineering, 1996, 2819:2-6.

Forman M L, Steel W H, Vanasse G A. Correction of Asymmetric Interferograms Obtained in Fourier Spectroscopy[J]. Josa, 1966, 56(1):59-61.

Mertz L. Auxiliary computation for Fourier spectrometry[J]. Infrared Physics, 1967, 7(1):17-23.

Sanderson R B, Bell E E. Multiplicative correction of phase errors in fourier spectroscopy.[J]. Applied Optics, 1973, 12(2):266.

Topping M Q, Pfeiffer J E, Sparks A W, et al. Advanced Airborne Hyperspectral Imaging System (AAHIS)[J]. Proceedings of SPIE - The International Society for Optical Engineering, 2002:1-11.

Wurtz R E, Blaisouellette S, Cook K H, et al. Visible Imaging Fourier Transform Spectrometer: Design and Calibration[J]. Proceedings of SPIE - The International Society for Optical Engineering, 2002, 4841:1067-1077. 